\title{
Real-time interferometric characterization of a polyvinyl alcohol based photopolymer at the zero spatial frequency limit
}

\author{
S. Gallego, ${ }^{1}$ A. Márquez, ${ }^{1}$ D. Méndez, ${ }^{1}$ C. Neipp, ${ }^{1}$ M. Ortuño,,${ }^{1, \star}$ M. Álvarez, ${ }^{1}$ E. Fernandez, ${ }^{2}$ \\ and A. Beléndez ${ }^{1}$ \\ ${ }^{1}$ Departamento de Física, Ingeniería de Sistemas, y Teoría de la Señal, Universidad de Alicante, Spain \\ 2Departamento Interuniversitario de Óptica, Universidad de Alicante, Spain \\ *Corresponding author: mos@ua.es
}

Received 1 June 2007; revised 10 September 2007; accepted 11 September 2007;

posted 11 September 2007 (Doc. ID 83649); published 16 October 2007

\begin{abstract}
We characterize the optical modulation properties of a polyvinyl alcohol/acrylamide (PVA/AA) photopolymer at the lowest end of recorded spatial frequencies. To achieve this goal we have constructed a double beam interferometer in combination with the setup to expose the recording material. This is a novel approach since usually holographic recording materials are only characterized at high spatial frequencies. Some benefits are provided by the approach we propose: a direct calculation of the properties of the material is possible, and on the other hand additional information can be obtained since the results are not influenced by diffusion processes. Furthermore, this characterization is needed to optimize the PVA/AA photopolymers for another range of applications, such as recording of diffractive optical elements, where very low spatial frequencies are recorded. Different PVA/AA compositions and layer thicknesses have been analyzed. We have found that, depending on the layer characteristics, we can achieve high values of the phase-shift modulation depth and enhance the sensitivity of the material. (c) 2007 Optical Society of America

OCIS codes: $160.0160,160.5470,160.4670$
\end{abstract}

\section{Introduction}

Photopolymer based recording materials have been widely studied for holographic applications [1-4]. Their good properties, such as high diffraction efficiencies, low noise, self-developing, easy preparation, high thickness, low cost, etc., make these holographic recording materials optimum candidates for many holographic devices such as holographic memories [5-7]. For these applications, good response of spatial frequencies, between 700 and 1500 lines $/ \mathrm{mm}$, is required. In particular, polyvinyl alcohol/acrylamide (PVA/AA) materials have been deeply analyzed in holography [8-10] and data storage [11]. Some theoretical models have been proposed [12-15] to study these applications and to predict the material behavior during the recording process. To obtain the values

0003-6935/07/307506-07\$15.00/0

(C) 2007 Optical Society of America for the modulation properties of the material, mainly the index modulation, nonlinear fitting of multiparametric models, such as the rigorous coupled-wave theory (RCWT) [8], is applied to the experimentally measured diffracted and/or transmitted intensity. Even though accurate values may be obtained with the holographic approach, it would also be very useful to obtain a direct measurement of the modulation properties of the material. It is also remarkable that the values obtained for the modulation properties at high spatial frequencies (more than 500 lines $/ \mathrm{mm}$ ) contain both the influence of photopolymerization reaction and diffusion of the components in the material between the exposed and unexposed zones [13]. Depending on the strength assumed in the models for the two processes (photopolymerization and diffusion), the values for the material parameters may largely vary. This is partly the reason for the huge differences in the values of the characteristic parameters provided by different research teams [16-20] 
for very similar compounds. Therefore, it would be a clear advantage to have some technique at hand so as to measure the characteristics of the material isolating one of the two processes.

In the last 10 years, some studies have been carried out dealing with the recording of very low spatial frequencies (less than 10 lines $/ \mathrm{mm}$ ) in PVA/AA based layers. In [21] a contact-copying process was used to transfer low spatial frequency diffractive optical elements (DOEs) from binary amplitude masks [22] onto the photopolymer, with good results. The same technique was used to analyze the suitability of a wide range of holographic recording materials at low spatial frequencies so as to register DOEs and optical correlation filters [23]. Recently, some works based on low spatial frequencies recording have been presented [24]. These authors analyze the surface profile observed in PVA/materials, concluding that the material swells at low spatial frequencies; another interesting work about the PVA/AA volume changes during exposure was presented by Kelly et al. [25], where they focused their attention on the influence of photopolymer components' refractive indexes.

We can conclude that very few studies have focused on the characteristics of holographic recording materials, and in particular of PVA/AA photopolymers at low spatial frequencies, even though there are some applications, such as DOEs generation, where low spatial frequencies are recorded. In addition, the modulation properties of these materials are in general very different at low spatial frequencies in comparison with the results at the typical range of holographic spatial frequencies. In the limit of zero frequency recording, i.e., uniform exposure of the photosensitive layer, no diffusion processes are produced (at least in the transverse direction along the layer). Taking all this into account, we propose in this paper a characterization technique especially designed to obtain information about the modulation properties of the material at the zero spatial frequency recording limit. Furthermore, as opposed to holographic characterization, the data obtained in this case are directly related to the physical properties of the material without the need of nonlinear fitting of multiparametric models. In particular we use an interferometric technique to characterize different layers' compositions based on PVA/AA photopolymers. This technique consists of a real time analysis of the phase shift as a function of exposure. First, we have studied thin layers ( $~ 80 \mu \mathrm{m}$ thick) with the basic chemical composition using only one monomer (AA); second, we have analyzed the inclusion of a cross-linker monomer $\left[N, N^{\prime}\right.$ methylenebisacrylamide (BMA)]. This monomer is used to achieve higher values of refractive index modulation and to obtain more stable gratings [3]. Work with thicker layers $(\sim 800 \mu \mathrm{m}$ thick) allows higher values of phase modulation; nevertheless, the chemical compositions must be changed (to decrease the noise produced by the high concentrations of monomer and dye [7]). We analyze the phase changes in these thick layers, focusing our attention in the importance of the cross-linker and the dye concentration.

\section{Experimental Procedure}

\section{A. Experimental Setup}

We have designed the experimental setup presented in Fig. 1 to measure the phase shift as a function of the exposure energy. The setup has two arms with an angular separation of $30^{\circ}$, one to expose the recording material, whereas the second arm is the interferometer used to measure, in real time, the phase shift. The recording material is perpendicularly oriented with respect to the interferometer axis in order to ease the analysis of the interferometric results: at an oblique incidence we should take into account both the Fresnel coefficients at the interface and the increase of distance in the propagation across the layer.

In the first arm, the exposure beam provided by a solid-state $\mathrm{Nd}-\mathrm{YVO}_{4}$ Verdi laser with a wavelength of $532 \mathrm{~nm}$ (at this wavelength the dye presents the maximum absorption) is expanded and collimated using a spatial filter and a lens, obtaining a beam with $1.5 \mathrm{~cm}$ of radius. A wave plate and a neutral filter (attenuator) are added before the spatial filter to control the orientation and the intensity of the linearly polarized beam produced by the $\mathrm{Nd}-\mathrm{YVO}_{4}$ laser. A polarizer $(\mathrm{P})$, with its transmission axis oriented along the vertical of the laboratory, is introduced to produce a beam with $\mathrm{TE}$ polarization incident onto the recording material. This incident beam forms an angle of $30^{\circ}$ with respect to the photopolymer layer. We adjust the laser power so that the exposure intensity that impinges on the layer is $0.4 \mathrm{~mW} / \mathrm{cm}^{2}$ (this is the value corrected from the Fresnel coefficient at the air-photopolymer interface at an incidence of $30^{\circ}$ for $\mathrm{TE}$ polarization). A half-opened diaphragm is used to leave an unexposed area in the photopolymer layer. In the interferometric arm, to generate the interferences pattern we use an $\mathrm{He}-\mathrm{Ne}$ laser, since the photopolymer does not present any absorption at $633 \mathrm{~nm}$. We have implemented a Young's fringes based two beams interferometer.

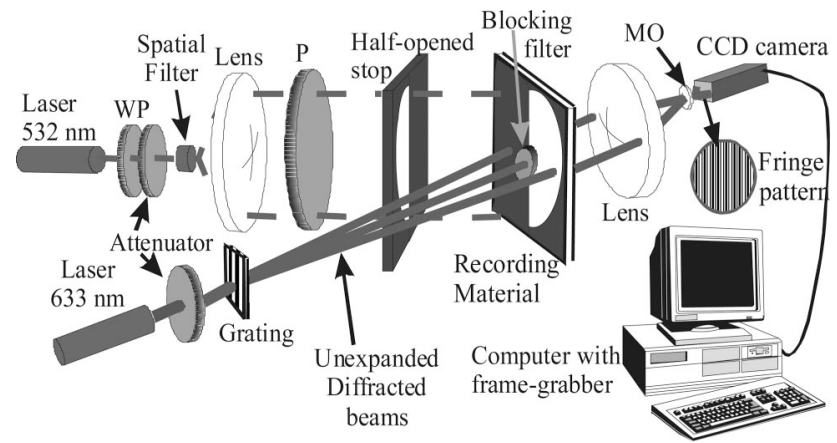

Fig. 1. Experimental setup. The recording material is exposed with the green laser beam $(\lambda=532 \mathrm{~nm})$ and the phase shift is measured with the red beam $(\lambda=633 \mathrm{~nm})$. $\mathrm{P}$ is the polarizer, WP is the wave plate, $\mathrm{MO}$ is the microscope objective. 


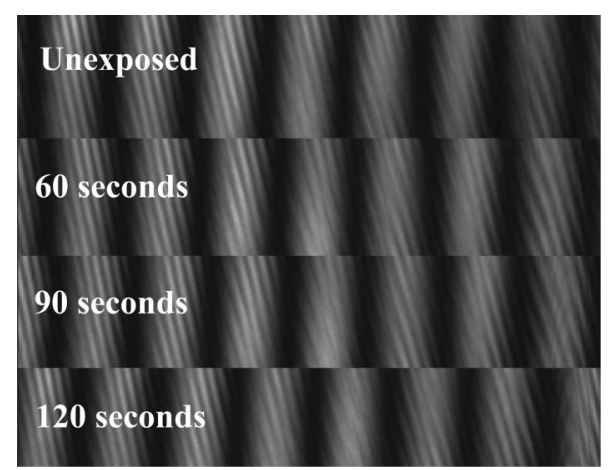

Fig. 2. Fringe pattern for different exposure times (before exposing the material, after 60, 90, and $120 \mathrm{~s}$, respectively). In particular, these images correspond to material 2 (see Table 1).

This interferometer has been successfully applied in the phase-shift characterization of liquid-crystal displays (LCDs) [26]. It shows good precision, and, due to its quasi-common-path architecture, is a robust setup, less sensitive to changing environmental conditions and simpler to construct than Mach-Zehnder type interferometers. We use a grating with a spatial frequency of 4 lines $/ \mathrm{mm}$ to generate a series of diffracted orders from the unexpanded $\mathrm{He}-\mathrm{Ne}$ beam; we block all the orders except -1 and +1 . One of the two orders impinges on the exposed zone (illuminated by the $\mathrm{Nd}-\mathrm{YVO}_{4}$ laser) and the other one impinges on the nonexposed zone. The distance between the two orders is approximately $1 \mathrm{~cm}$, so as to eliminate the influence of the monomer diffusion in the polymerization process. Once the two orders have propagated throughout the photopolymer, a lens is used to make them interfere. A microscope objective is used to amplify the interference pattern onto a CCD camera, Hamamatsu model C5403. This pattern is captured in real time as a function of exposure at specific time intervals, then is digitized and transferred to a personal computer by a frame-grabber, Matrox model Meteor.

In Fig. 2 we show as an example the interference fringes captured at four different exposure times. We see how the fringes shift while the exposure time increases. The patterns show a very good visibility, a fact that implies the amplitude transmission does not vary with the exposure. Actually the material can be considered transparent to the $633 \mathrm{~nm}$ wavelength. Therefore, with this setup it is possible to plot the fringes moving as a function of the exposure time during the polymerization process and without the influence of monomer diffusion. In this sense, we have verified that once we stop the exposition, the shift in the interference fringes freezes. This indicates that the measurements taken in this setup are not affected by diffusion processes.

Once the interference pattern has been stored, we measure the shift with respect to the initial pattern obtained for the unexposed layer. To increase the accuracy in this calculation, we cross correlate the different interference patterns with respect to the unexposed ones. The cross correlation produces a clear peak. The location of this peak for each exposure with respect to the center of the image is equal to the shift in the fringe pattern. A full fringe shift is equal to a $2 \pi$ rad variation in the phase shift. In this experiment, the phase shift is directly related to the index modulation of the material. This simple and direct relation is not possible in the case of the holographic characterization, where multi-parametric diffusion models are used.

\section{B. Photopolymer Layers}

The PVA/AA formulations contain a dye, a cosensitizer that is triethanolamine, one or two monomers (AA and or not BMA), and a polymer (PVA). Let us introduce some basic details related to the design of the composition of PVA/AA photopolymer layers. Since the molecular weight of the PVA plays an important role in the monomer diffusion during the polymerization process [27], different types of PVA have been used in the literature. Moreover, different dyes and different triethanolamine (TEA) concentrations are normally used to obtain different material properties (TEA is a liquid at ambient temperature and plays an important role in the monomer and polymer diffusion during the polymerization process as well [28]).

In this paper, we consider PVA with a molecular weight of 130,000, and the TEA concentration is varied between 0.4 and $0.15 \mathrm{M}$ (see Table 1). The photopolymerizable solution is prepared mixing yellowish eosin (YE, the dye), together with a mixture of AA (the monomer) and TEA (the co-initiator), and adding the PVA (the binder). In this study the solutions are prepared using a conventional magnetic stirrer, under red light, and in standard laboratory conditions (temperature, pressure, relative humidity). The solutions are deposited, by gravity, over glasses (size $20 \mathrm{~cm} \times 40 \mathrm{~cm}$ ) in the case of thin layers and in polystyrene circular molds in the case of thick layers. Afterward the solutions are left in the dark to allow the water evaporation. When a high percentage of the water content has already evaporated [11], the

Table 1. Water Solutions Used to Obtain Photopolymerizable "Dry" Layers

\begin{tabular}{lccccc}
\hline \multicolumn{1}{c}{ Component } & Type 1 & Type 2 & Type 3 & Type 4 & Type 5 \\
\hline PVA $M_{w}=130,000$ & $8.5 \% \mathrm{w} / \mathrm{v}$ & $8.5 \% \mathrm{w} / \mathrm{v}$ & $3.30 \% \mathrm{w} / \mathrm{v}$ & $13.30 \% \mathrm{w} / \mathrm{v}$ & $13.30 \% \mathrm{w} / \mathrm{v}$ \\
TEA & $0.4 \mathrm{M}$ & $0.4 \mathrm{M}$ & $0.15 \mathrm{M}$ & $0.15 \mathrm{M}$ & $0.15 \mathrm{M}$ \\
YE & $2.5 \times 10^{-4} \mathrm{M}$ & $2.5 \times 10^{-4} \mathrm{M}$ & $9.0 \times 10^{-5} \mathrm{M}$ & $9.0 \times 10^{-5} \mathrm{M}$ & $4.5 \times 10^{-5} \mathrm{M}$ \\
AA & $0.45 \mathrm{M}$ & $0.40 \mathrm{M}$ & $0.34 \mathrm{M}$ & $0.31 \mathrm{M}$ & $0.31 \mathrm{M}$ \\
BMA & None & $0.05 \mathrm{M}$ & None & $0.04 \mathrm{M}$ & $0.04 \mathrm{M}$ \\
\hline
\end{tabular}


thin material is cut into squares using a glass cutter; and the thick "dry" material is removed from the mold, cut into squares, and deposited, without the need of adhesive, onto the surface of glass plates (measuring $6.5 \mathrm{~cm} \times 6.5 \mathrm{~cm}$ ). The water solutions used in this paper are presented in Table 1. Composition types 1 and 2 are used to obtain thin layers, with thickness between 60 and $120 \mu \mathrm{m}$; in the case of composition type 2 the BMA is included in the solution [29]. To obtain thick layers, with a thickness between 700 and $1000 \mu \mathrm{m}$, we need to increase the PVA concentration (achieving more consistent layers). Solution type 3 has no BMA while solution type 4 has BMA as a cross-linker. In solution type 5 the dye concentration has been reduced $50 \%$ to obtain more penetration of the green beam inside the material.

\section{Results and Discussion}

In this section we present the experimental data obtained for different material compositions and different thicknesses. It is interesting to note that the material presents very different behavior for each composition. For instance, when cross-linker monomer is added, the polymerization rates increase drastically [29]. The size of the error bars included in the figures indicates the repeatability or our experiments. This repeatability is higher in thin layers and decreases when there are variations of the humidity and temperature in our laboratory during the drying process.

\section{A. Thin Layers}

Let us begin the analysis with the basic material composition (type 1). In Fig. 3 we show the phase shift between the bright and dark zones as a function of the exposure time for material type 1 ; these layers have a thickness of $70 \mu \mathrm{m}$. We can see that after an exposure of $400 \mathrm{~s}\left(160 \mathrm{~mJ} / \mathrm{cm}^{2}\right)$, the material is in the saturation region and no more changes can be observed: it is possible to assume that the polymerization process is finished. The saturation energy value is in the range obtained when characterizing the material at high spatial frequencies $\left(\sim 120 \mathrm{~mJ} / \mathrm{cm}^{2}\right)$. The maximum phase-shift value is approximately $192^{\circ}$; this value is sufficient in-order to store binary-

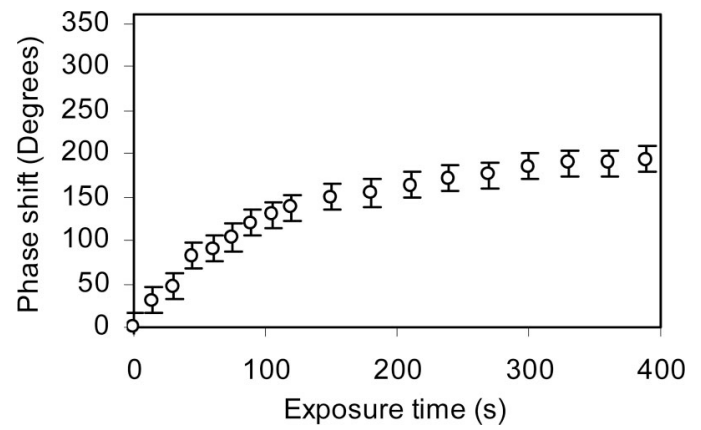

Fig. 3. Phase shift as a function of the exposure time for layers type 1 and $70 \mu \mathrm{m}$ thick.
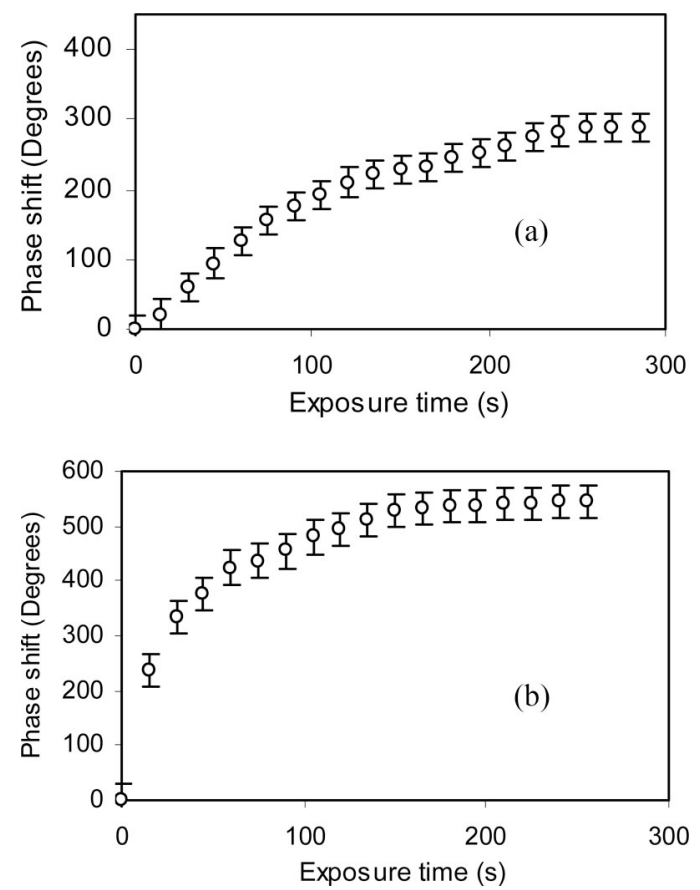

Fig. 4. Phase shift as a function of the exposure time for layers type 2, (a) for $70 \mu \mathrm{m}$ thick and (b) for $115 \mu \mathrm{m}$ thick.

phase DOEs but it is too small to record nonbinary DOEs, as diffractive lenses, blazed gratings, or kinoforms, where a $360^{\circ}$ phase-shift depth is generally required [30].

To achieve higher values of phase shift, there are two possibilities: to increase the thickness or to increase the refractive index modulation. To increase the refractive index, we can use cross-linker monomers such as BMA. In Fig. 4(a) we present the results provided for material type 2 (for the same thickness of material type 1, $70 \mu \mathrm{m}$ ). In Fig. 4(b) we present the results provided for material type 2 with a thickness of $115 \mu \mathrm{m}$. It is possible to observe that if we use BMA in the chemical material composition, we obtain higher values of the phase shift. Particularly, in Fig. 4(b), we achieve phase shifts significantly larger than $360^{\circ}$; that is, we can use this material to store nonbinary DOEs as lenses. The only important disavantage is the nonlinearity between exposure time and phase shift. Moreover, it is important to note the difference between the saturation exposure times for material type 1 (without BMA), shown in Fig. 1, and type 2 (with BMA). Material type 1 presents saturation exposure times of approximately $300 \mathrm{~s}$ whereas in the case of material type 2 these times are approximately $200 \mathrm{~s}$. This effect clearly shows the increase in the polymerization rate when BMA is present [29].

\section{B. Thick Layers}

Due to their high dynamic range, the main application of the photopolymer-based thick layers is the development of holographic memories [31]. In this paper, this high dynamic range may possibly be translated into a high phase-shift depth, attractive for DOE generation. It is also interesting to analyze 

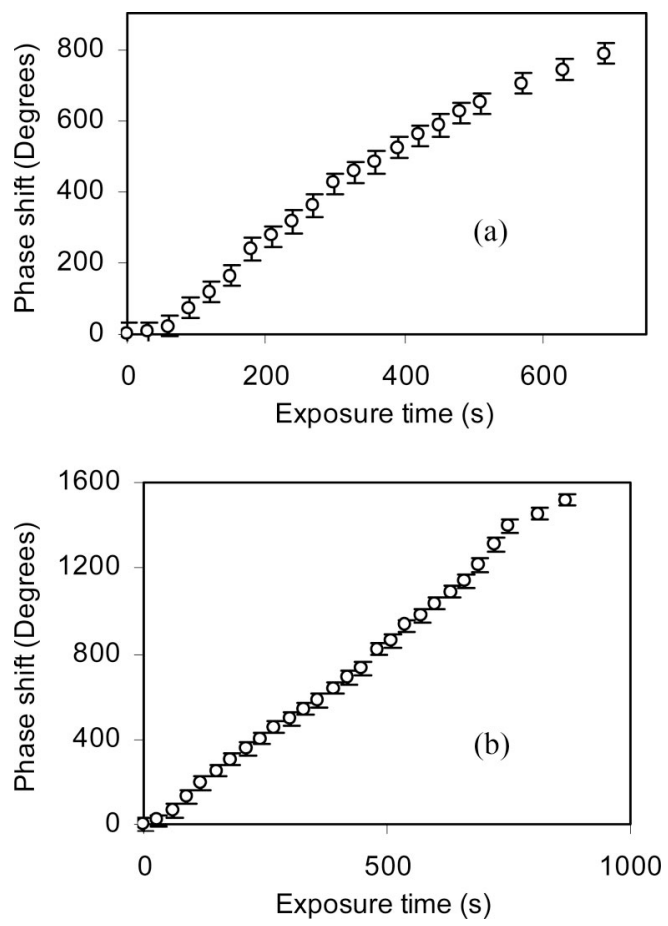

Fig. 5. Phase shift as a function of the exposure time for $800 \mu \mathrm{m}$ layers, (a) without BMA and (b) with BMA.

the phase-shift profile (linearity, saturation behavior) versus the exposure time in these thick layers in comparison with the results for thin layers. The chemical composition for these thick layers requires low dye and monomer concentrations, which produces a slower polymerization rate. Since we use low exposure intensity and the polymerization rate is very small in our experiments, it is possible to observe the inhibition period [32-34] of these materials. Figures 5(a) and 5(b) show the phase shift as a function of exposure time for chemical compositions type 3 and type 4 (without and with BMA, respectively), and for a thickness of $800 \mu \mathrm{m}$. In this sense, it is possible to see that the inhibition time decreases when BMA is present. In addition to this, we observe the same effect analyzed in Subsection 3.A: BMA permits obtaining higher values of phase shift and higher material sensitivity (that is, the curve's slope is larger). Besides, we clearly see the linearity between phase shift and exposure time. The reason is that, even though the exposure time is very high (more than $600 \mathrm{~s}$ ) the profile does not show a saturation performance. Phase-shift values larger than $1000^{\circ}$ may be possible for these very thick layers.

\section{Different Dye Concentration}

One of the problems analyzed in some papers using composition types 3 and 4 ( $800 \mu \mathrm{m}$ thick) is the high attenuation of the light inside the material [35-37] (before exposure, the layer transmittance for a wavelength of $532 \mathrm{~nm}$ is approximately $0.05 \%$ ). This means that polymerization only takes place in the first part of the material, so there are differences between the physical thickness of the layer and the

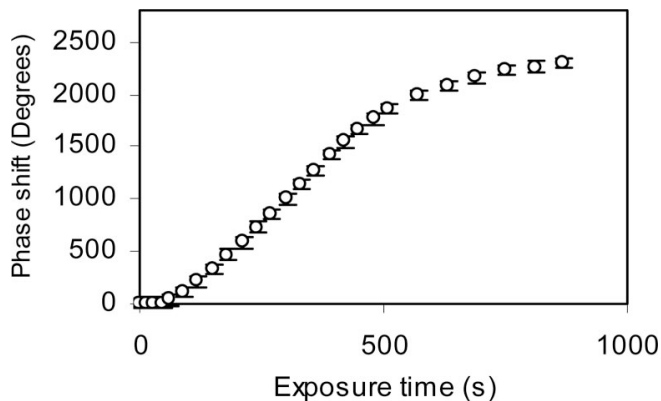

Fig. 6. Phase shift as a function of the exposure time for layers type 5 .

effective optical thickness of the elements stored [36]. To reduce the material absorption and to use all the physical thickness, we have characterized material type 5 , where the dye concentration has been reduced to 50\%. The experimental data are plotted in Fig. 6, where it is possible to see that the inhibition period is increased for this chemical composition. Furthermore, the energetic sensitivity of this material is very high: $\sim 2000^{\circ}$ of phase shift is achieved in only $500 \mathrm{~s}$. This effect implies that the effective optical thickness of the layer is higher. In this sense, it is possible to see, after the first $80 \mathrm{~s}$ (the inhibition time), that there is a linear relation between phase shift and exposure time until $500 \mathrm{~s}$, where the material reaches the saturation region.

Eventually, once we have characterized the different layers, it is important to remark that the huge phase shift between the bright and dark zones cannot only be explained by a refractive index modulation, that is, a certain degree of thickness variation between the bright and dark regions should clearly contribute to the phase shift. If we assume that there is no thickness variation, the saturation refractive index modulation should be approximately $5 \times 10^{-3}$ for material type $1,8 \times 10^{-3}$ for material type 2 , and $3.5 \times 10^{-2}$ for material type 5 . Taking into account these results, the thin layers' values are $30 \%$ higher than the typical values of refractive index modulation obtained in the holographic range, where the spatial frequencies are approximately 1000 lines $/ \mathrm{mm}$. Furthermore, in the case of the holographic high spatial frequencies, there is an important contribution of free monomer coming from the nonexposed zones (monomer diffusion plays an important role for high spatial frequencies), which contributes to increasing the refractive index modulation. In the case of thick layers, results indicate even a stronger thickness shift since the values of the refractive index modulation obtained are $250 \%$ higher compared with the ones obtained for holographic gratings in this kind of layers. These results suggest that PVA/AA based materials present a swelling or a shrinkage during the polymerization process in the experiments carried out. This effect is more important for thick layers. In this sense it is well-known that volume shrinkage acts during the homogeneous polymerization of polymers $[38,39]$. 


\section{Conclusions}

In this paper we have characterized the optical modulation properties of five interesting chemical compositions of PVA/AA based materials and for a very wide range of thicknesses (ranging from 75 to $800 \mu \mathrm{m}$ ). A novel technique based on a Young's based double beam interferometer is proposed, which allows obtaining real-time direct values for the index modulation of the recording material as a function of exposure. The characterization technique proposed does not depend on monomer diffusion processes, since a uniform exposure (zero frequency) is recorded onto the material. From the point of view of the phase-shift range depth, we have demonstrated the capability of these materials to store phase elements under red illumination. We have achieved large phase shifts between exposed and nonexposed zones exceeding $360^{\circ}$. Values for the sensitivity, linearity, inhibition times, and possible influence of thickness shift have been discussed.

This work was supported by the Ministerio de Edicación y Ciencia (Spain) under projects FIS200505881-C02-01 and FIS2005-05881-C02-02 and by the Generalitat Valenciana, under project GV06-007.

\section{References}

1. A. Pu and D. Psaltis, "High-density recording in photopolymerbased holographic three-dimensional disks," Appl. Opt. 35, 2389-2398 (1996).

2. Y. Tomita, K. Furushima, K. Ochi, K. Ishizu, A. Tanaka, M. Ozawa, M. Hidaka, and K. Chikama, "Organic nanoparticle (hyperbranched polymer)-dispersed photopolymers for volume holographic storage," Appl. Phys. Lett. 88, 071103 (2006).

3. A. Márquez, C. Neipp, A. Beléndez, S. Gallego, M. Ortuño, and I. Pascual, "Edge-enhanced imaging with polyvinyl alcohol/ acrylamide photopolymer gratings," Opt. Lett. 28, 1510-1512 (2003).

4. S. M. Schultz, E. N. Glytsis, and T. K. Gaylord, "Design of high-efficiency volume gratings couplers for line focusing," Appl. Opt. 37, 2278-2287 (1998).

5. D. A. Waldman, C. J. Butler, and D. H. Raguin, "CROP holographic storage media for optical data storage greater than $100 \mathrm{bits} / \mu \mathrm{m}^{2}$," Proc. SPIE 5216, 10-25 (2003).

6. W. L. Wilson, K. R. Curtis, K. Anderson, M. C. Tackitt, A. J. Hill, M. Pane, C. Stanhope, T. Earhart, W. Loechel, C. Bergman, K. Wolfgang, C. Shuman, G. Hertrich, K. Parris, K. Malang, B. Riley, and M. Ayer, "Realization of highperformance holographic data storage: the InPhase Technologies demonstration platform," Proc. SPIE 5216, 178-191 (2003).

7. H. J. Coufal, D. Psaltis, and G. T. Sincerbox, eds., Holographic Data Storage, Springer Series in Optical Sciences (SpringerVerlag, 2000).

8. C. Neipp, A. Beléndez, S. Gallego, M. Ortuño, I. Pascual, and J. T. Sheridan, "Angular responses of the first and second diffracted orders in transmission diffraction grating recorded on photopolymer material," Opt. Express 11, 1835-1843 (2003).

9. J. V. Kelly, F. T. O’Neill, C. Neipp, S. Gallego, M. Ortuño, and J. T. Sheridan, "Holographic photopolymer materials: nonlocal polymerization-driven diffusion under nonideal kinetic conditions," J. Opt. Soc. Am. B 22, 407-416 (2005).

10. S. Martin, C. A. Feely, and V. Toal, "Holographic recording characteristics of an acrylamide-based photopolymer," Appl. Opt. 36, 5757-5768 (1994).
11. M. Ortuño, S. Gallego, C. García, C. Neipp, A. Beléndez, and I. Pascual, "Optimization of a $1 \mathrm{~mm}$ thick PVA/acrylamide recording material to obtain holographic memories: method of preparation and holographic properties," Appl. Phys. B 76, 851-857 (2003).

12. R. R. Adhami, D. J. Lanteigne, and D. A. Gregory, "Photopolymer hologram formation theory," Microwave Opt. Technol. Lett. 4, 106-109 (1991).

13. G. Zhao and P. Mouroulis, "Diffusion model of hologram formation in dry photopolymer materials," J. Mod. Opt. 41, 19291939 (1994).

14. S. Piazzolla and B. K. Jenkins, "First-harmonic diffusion model for holographic grating formation in photopolymers," J. Opt. Soc. Am. B 17, 1147-1157 (2000).

15. J. T. Sheridan and J. R. Lawrence, "Nonlocal-response diffusion model of holographic recording in photopolymer," J. Opt. Soc. Am. A 17, 1008-1014 (2000).

16. F. T. O’Neill, J. R. Lawrence, and J. T. Sheridan, “Comparison of holographic recording materials by use of analytical nonlocal diffusion model," Appl. Opt. 41, 845-852 (2002).

17. S. Blaya, L. Carretero, R. F. Madrigal, M. Ulibarrena, P. Acebal, and A. Fimia, "Photopolymerization model for holographic gratings formation in photopolymers," Appl. Phys. B 77, 639-662 (2003).

18. J. V. Kelly, M. R. Gleeson, C. E. Close, F. T. O'Neill, J. T. Sheridan, S. Gallego, and C. Neipp, "Temporal analysis of grating formation in photopolymer using the nonlocal polymerization-driven diffusion model," Opt. Express 13, 6990-7004 (2005).

19. S. Gallego, C. Neipp, M. Ortuño, A. Márquez, A. Beléndez, and I. Pascual, "Diffusion-based model to predict the conservation of gratings recorded in poly(vinyl alcohol): acrylamide photopolymer," Appl. Opt. 42, 5839-5845 (2003).

20. I. Naydenova, R. Jallapuram, R. Howard, S. Martin, and V. Toal, "Investigation of the diffusion processing in selfprocessing acrylamide-based photopolymer system," Appl. Opt. 43, 2900-2905 (2004).

21. I. Pascual, A. Márquez, A. Beléndez, A. Firnia, J. Campos, and M. J. Yzuel, "Copying low spatial frequency diffraction gratings in photopolymer as phase holograms," J. Mod. Opt. 47, 1089-1097 (2000).

22. A. Márquez, J. Campos, M. J. Yzuel, I. Pascual, A. Fimia, and A. Beléndez, "Production of computer-generated phase holograms using graphic devices: application to correlation filters," Opt. Eng. 39, 1612-1619 (2000).

23. A. Márquez, C. Neipp, A. Beléndez, J. Campos, I. Pascual, M. J. Yzuel, and A. Fimia, "Low spatial frequency characterization of holographic recording materials applied to correlation,” J. Opt. A, Pure Appl. Opt. 5, S175-S182 (2003).

24. I. Naydenova, E. Mihaylova, S. Martin, and V. Toal, "Holographic patterning of acrylamide-based photopolymer surface," Opt. Express 13, 4878-4889 (2005).

25. J. V. Kelly, M. R. Gleeson, C. E. Close, and J. T. Sheridan, "Nonlocal polymerization driven diffusion model: volume changes during grating formation," Proc. SPIE 6335, 63350Q (2006).

26. A. Bergeron, J. Gauvin, F. Gagnon, D. Gingras, H. H. Arsenault, and M. Doucet, "Phase calibration and applications of a liquid-crystal spatial light modulator," Appl. Opt. 34, 5133-5139 (1995).

27. V. Weiss, E. Millul, and A. A. Friesem, "Improvements in holographic photopolymers at Weizmann Institute of Science," SPIE Int. Tech. Group Newsletter: Opt. Inf. Syst. (SPIE, 2004), Vol. 1, p. 3.

28. S. Gallego, M. Ortuño, C. Neipp, A. Márquez, A. Beléndez, and I. Pascual, "Characterization of polyvinyl alcohol-acrylamide holographic memories with a first-harmonic diffusion model," Appl. Opt. 44, 6205-6210 (2005). 
29. C. Neipp, S. Gallego, M. Ortuño, A. Márquez, A. Beléndez, and I. Pascual, "Characterization of a PVA/acrylamide photopolymer. Influence of a cross-linking monomer in the final characteristics of the hologram," Opt. Commun. 224, 27-34 (2003).

30. D. C. O'Shea, T. J. Suleski, A. D. Kathman, and D. W. Prather, eds., Diffractive Optics: Design, Fabrication, and Test (SPIE, 2004).

31. E. Fernández, C. García, M. Ortuño, S. Gallego, A. Beléndez, and I. Pascual, "Optimization of a thick PVA/acrylamide photopolymer for data storage using a combination of angular and peristrophic holographic multiplexing," Appl. Opt. 45, 76617666 (2006).

32. P. J. Flory, Principles of Polymer Chemistry (Cornell U. Press, 1953), pp. 161-177.

33. M. Ortuño, S. Gallego, C. García, C. Neipp, and I. Pascual, "Holographic characteristics of a $1 \mathrm{~mm}$ thick photopolymer to be used in holographic memories," Appl. Opt. 42, 7008-7012 (2003).

34. M. R. Gleeson, J. V. Kelly, C. E. Close, F. T. O’Neill, and J. T. Sheridan, "The impact of inhibition processes during grating formation in photopolymer materials," Proc. SPIE 5827, 232243 (2005).
35. C. Neipp, J. T. Sheridan, S. Gallego, M. Ortuño, I. Pascual, and A. Beléndez, "Effect of a depth attenuated refractive index profile in the angular responses of the efficiency of higher orders in volume gratings recorded in a PVA/acrylamide photopolymer," Opt. Commun. 233, 311-322 (2004).

36. S. Gallego, M. Ortuño, C. Neipp, A. Márquez, A. Beléndez, I. Pascual, J. V. Kelly, and J. T. Sheridan, "Physical and effective optical thickness of holographic diffraction gratings recorded in photopolymers," Opt. Express 13, 1939-1950 (2005).

37. S. Gallego, M. Ortuño, C. Neipp, A. Márquez, A. Beléndez, I. Pascual, J. V. Kelly, and J. T. Sheridan, "3 dimensional analysis of holographic photopolymers based memories," Opt. Express 13, 3543-3554 (2005).

38. J. E. Dietz and N. A. Peppas, "Reaction kinetics and chemical changes during polymerization of multifunctional (meth)acrylates for the production of highly crosslinked polymers used in information storage systems," Polymer 38, 3767-3781 (1997).

39. T. Endo and F. Sanda, "Ring-opening polymerization, anionic (with expansion in volume)," in Polymeric Materials Encyclopedia, J. C. Salamone, ed. (CRC, 1996), Vol. 10, pp. 7550-7553. 